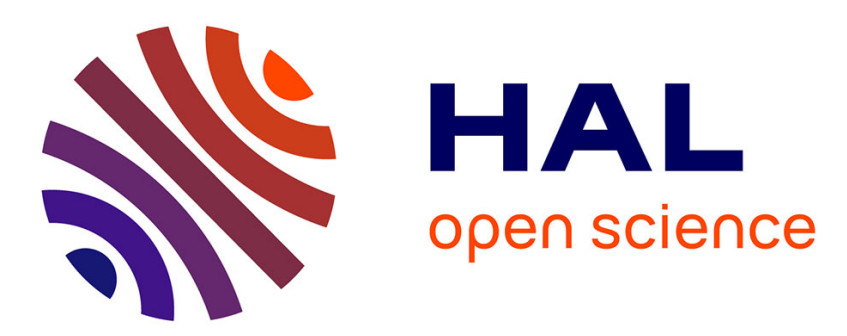

\title{
The type II secretion system - a dynamic fiber assembly nanomachine
}

\author{
Manuel Campos, David Cisneros, Mangayarkarasi Nivaskumar, Olivera \\ Francetic
}

\section{- To cite this version: \\ Manuel Campos, David Cisneros, Mangayarkarasi Nivaskumar, Olivera Francetic. The type II se- cretion system - a dynamic fiber assembly nanomachine. Research in Microbiology, 2013, 164 (6), pp.545-555. 10.1016/j.resmic.2013.03.013 . hal-02351800}

\section{HAL Id: hal-02351800 https://hal.science/hal-02351800}

Submitted on 3 Jun 2021

HAL is a multi-disciplinary open access archive for the deposit and dissemination of scientific research documents, whether they are published or not. The documents may come from teaching and research institutions in France or abroad, or from public or private research centers.
L'archive ouverte pluridisciplinaire HAL, est destinée au dépôt et à la diffusion de documents scientifiques de niveau recherche, publiés ou non, émanant des établissements d'enseignement et de recherche français ou étrangers, des laboratoires publics ou privés. 
3 Manuel Campos ${ }^{\mathrm{a}, \mathrm{c}}$, David A. Cisneros ${ }^{\mathrm{b}}$, Mangayarkarasi Nivaskumar ${ }^{\mathrm{a}}$ and Olivera Francetic

4 Institut Pasteur, Molecular Genetics Unit, Department of Microbiology, 75015 Paris, France.

CNRS ERL3526, 75724 Paris, France.

${ }^{\text {b} P r e s e n t ~ a d d r e s s: ~ R e s e a r c h ~ I n s t i t u t e ~ o f ~ M o l e c u l a r ~ P a t h o l o g y ~(I M P), ~ D r . ~ B o h r-G a s s e ~ 7, ~ A-1030 ~}$

8 Vienna, Austria and Max F. Perutz Laboratories (MFPL), University of Vienna, Dr. BohrGasse 9, A-1030 Vienna, Austria

'Present address: Yale University School of Medicine, New Haven, CT, USA

Abstract (100 w)

Type II secretion systems (T2SSs) share common origins and structure with archaeal flagella

14 (archaella) and pili, bacterial competence systems and type IV pili. All of these systems use a conserved ATP-powered machinery to assemble helical fibers that are anchored in the plasma membrane. The T2SSs assemble pseudopili, periplasmic filaments that promote extracellular secretion of folded periplasmic proteins. Comparative analysis of T2SSs and related fiber assembly nanomachines might provide important clues on their functional specificities and dynamics. This review focuses on recent developments in the study of pseudopilus structure and biogenesis, and discusses mechanistic models of pseudopilus function in protein secretion. 


\section{Introduction}

The type 2 secretion systems (T2SSs) translocate folded proteins from the periplasm of Gramnegative bacteria across the outer membrane via a large channel that remains closed in the resting state (reviewed by P. Howard in this issue). Upon secretion, the exoprotein substrates are released or exposed on the bacterial surface (Rondelet and Condemine, this issue) to perform diverse functions in macromolecule degradation, adhesion, electron transport or pathogenesis. The remarkable progress made in the study of T2SS structure and organization during the past decade has been described in detail in several recent reviews (Douzi et al., 2012, Korotkov et al., 2012, McLaughlin et al., 2012). This review focuses on the core function of T2SS - the assembly of periplasmic filaments called the pseudopili, which play an essential but poorly understood function in promoting the protein transport.

The T2SSs belong to an ancient and widespread superfamily of membrane nanomachines that specialize in the assembly of fibers built with subunits localized in the inner membrane. This highly successful and versatile molecular device is found in all prokaryotes, suggesting its ancient evolutionary origin. In archaea these systems form the base of archaella (formerly archaeal flagella) (Jarrel and Albers, 2012), type IV-like pili and bindosomes, the carbohydrate "capture" systems. In bacteria, beside the natural competence systems and T2SSs, they are also found in the form of type IV pili (T4P), long flexible fibers extending from the bacterial surface. T4P are involved in bacterial motility over solid substrates called twitching motility, and in other functions including adhesion, DNA uptake, aggregation, biofilm formation, signaling and protein transport (Pelicic, 2008).

All these diverse machineries have a common basic function - the assembly of helical fibers from the plasma membrane localized pilin subunits. The pilin transmembrane (TM) segments are highly conserved and comprise at the N-terminus a positively charged signal 
anchor followed by a Gly or Ala residue, the cleavage site for a specific processing enzyme, the prepilin peptidase. The hydrophobic segment extends into a long $\alpha$-helical stem that is shielded on one side by a globular domain composed of 4 to 5 anti-parallel $\beta$-strands separated by different conserved or variable loop regions. Globular pilin domains are exposed on the surface of assembled fibers with their variable sequence providing the fibers with diverse surface and binding properties.

The striking primary sequence conservation of TM $\alpha$-helical regions is the signature allowing unambiguous identification of fiber assembly systems in bacterial and archaeal genomes (Albers and Pohlschroder, 2009). Such sequence conservation suggests that a strong selective pressure shaped these segments, which play crucial roles in several steps of the fiber biogenesis pathway (Fig. 1). Their hydrophobicity is essential for interactions with the signal recognition particle (Fig. 1a) and for co-translational targeting to the Sec translocase that promotes pilin insertion in the membrane in an N-in - C-out orientation (Fig. 1b) (Arts et al., 2007b, Francetic et al., 2007). The specific prepilin peptidase removes the positively charged signal anchor after the conserved Gly residue (Fig. 1c), and, in T4a pili and T2SS transfers a methyl group to the new N-terminal residue. In the following, still poorly understood steps of fiber biogenesis, the TM segment might mediate interactions with the conserved fiber assembly factors in the membrane, including the polytopic IM protein of the PulF/PilC family (Fig. 1d). Finally, the TM segments make contacts with neighboring pilins during fiber assembly and form the hydrophobic core of assembled filaments (Fig. 1e).

\section{T2SS and fiber assembly nanomachines}

In the T2SS, between 12 and 15 genes are required for protein secretion. Their organization is similar in different bacterial groups (Douzi et al., 2012). In most species these 
genes form a single operon gspCDEFGHIJKLMNO (for general secretion pathway) (Fig. 2a), with different additional genes required for the assembly and targeting of the secretin channel GspD subunits. Each gsp gene is essential for protein secretion, with the exception of gspN, which is also absent from many bacteria. While this secretion-null phenotype did not favor the genetic analysis of T2SSs, biochemical dissection of the system led to the current model of T2SS organization in the bacterial envelope. Furthermore, the three-dimensional structure of most soluble domains of T2SS components has been determined (McLaughlin et al., 2012, Korotkov et al., 2012).

The functional studies of the T2SS have benefited from the comparative analysis with type IV pili (T4P), the most prominent and best-studied members of the helical fiber assembly nanomachine family. These thin and flexible filaments are found on the surface of many Gramnegative and some Gram-positive bacteria (Pelicic, 2008). In Pseudomonas aeruginosa, the core genes essential for TP4 biogenesis are organized in an operon pilABCD, encoding the major pilin subunit PilA, a hexameric ATPase PilB, a polytopic membrane protein PilC and the prepilin peptidase PilD. This basic fiber assembly module is found in all related systems, including the T2SS, suggesting that pilus assembly and protein secretion, as well as natural competence, share a common molecular mechanism (Hobbs and Mattick, 1993).

Five proteins contain an N-terminal prepilin peptidase cleavage site - the highly abundant (major) GspG and low-abundance (minor) pseudopilins GspH, I, J and K. The presence of these components and their processing and $\mathrm{N}$-methylation by the prepilin peptidase GspO suggested that T2SSs assemble short periplasmic fibers (Strom and Lory, 1991, Pugsley, 1993). These initially hypothetical structures, the pseudopili, could be visualized upon overexpression of the pul genes encoding the Klebsiella oxytoca T2SS (Sauvonnet et al., 2000b). Pseudopilus surface assembly allowed the biochemical and structural characterization of these fibers and provided an additional phenotype for genetic analysis of the T2SS. Fiber 
assembly is not specific to the Pul system: at least two others, the chitinase-specific Gsp T2SS from Escherichia coli (Vignon et al., 2003) and the P. aeruginosa Xcp system (Durand et al., 2003) also show this property. In contrast, the Hxc system in P. aeruginosa does not assemble surface pili under these conditions and seems to belong to a distinct T2SS subclass based on phylogenetic studies (Durand et al., 2011). While protein secretion strictly requires the presence of all T2SS components except GspB and GspN, pilus assembly, studied under overexpression conditions, does not require minor pseudopilins GpsH, GspI, GspJ and GspK, and the inner membrane component GspM (Fig. 2) (Durand et al., 2005). GspI has been implicated in pseudopilus assembly initiation since reduced amount of pili is found in $g s p I$ $(x c p V)$ mutants (Sauvonnet et al., 2000b, Durand et al., 2005). Similar to T4P in Neisseria spp. (Wolfgang et al., 2000, Carbonnelle et al., 2006), the secretin pore in the outer membrane is not required for fiber assembly but rather for the surface presentation of assembled fibers. In the absence of $K$. oxytoca PulD, PulG pili are assembled in the periplasm (Vignon et al., 2003, Cisneros et al., 2011).

\section{The major pseudopilins and T2SS pili}

Only major pseudopilins can form long surface polymers, while minor pseudopilins fail to form such fibers even when overproduced (Durand et al., 2005). Although surface pili are considered as an overproduction artifact, they facilitated the pseudopilus analysis using biochemical approaches, fluorescence and electron microscopy (EM). When observed by negative staining EM, pseudopili are highly similar to T4a pili, thin $(\approx 6 \mathrm{~nm})$, flexible and with bundling propensity, which might result from the EM sample preparation (Sauvonnet et al., 2000a, Durand et al., 2003). EM analysis of PulG pili shows a helical subunit arrangement: each subunit is displaced relative to its neighbor in the fiber by a vertical shift (axial rise) of $10.4 \AA$ 
and a rotational (twist) angle of $84.7^{\circ}$ (Kohler et al., 2004), values similar to those found in T4a pili (Craig et al., 2006). X-ray crystallography of major pseudopilins showed a typical pilin fold, with a long alpha-helical stem and an antiparallel 3-strand beta-sheet forming the globular domain (Kohler et al., 2004, Korotkov et al., 2009). Interestingly, the C-terminal pseudopilin domain is folded around a $\mathrm{Ca}^{2+}$ ion, forming a stable and well-structured loop (Korotkov et al., 2009), which is also apparent in the NMR structure of major pseudopilin $\mathrm{XcpT}^{\mathrm{GspG}}$ from $P$. aeruginosa (Alphonse et al., 2010). In $V$. cholerae the $\mathrm{Ca}^{2+}$ coordinating residues are essential for protein secretion (Korotkov et al., 2009), apparently without affecting the monomer stability. In the Pul T2SS, equivalent mutations reduce dramatically the levels of PulG, suggesting the involvement of $\mathrm{Ca}^{2+}$ in pseudopilin folding and stability (Campos et al., 2010). Interestingly, this loop has its counterpart in the variable loop of major T4a pilins that is stabilized by a disulfide bridge (Craig et al., 2006).

For T4a pili, a combination of cryo-EM and X-ray crystallography led to the first lowresolution models of these fibers (Craig et al., 2003). The first PulG pilus models, generated by a similar approach revealed the presence of deep groves on the fiber surface (Kohler et al., 2004). A prominent left-handed grove had been taken as evidence that PulG pili were organized as a left-handed helix (Kohler et al., 2004). However, the missing TM segments and the overall lack of detail precluded the use of this model for further structure-function analysis. The helical parameters (i.e. axial rise and rotation) from this study were used as constraints to build another series of PulG pilus models using a flexible ab initio approach (Campos et al., 2011). The 1000 models generated independently converged into a cluster of 200 highly similar right-handed helix models. The analysis of inter-protomer distances in this model cluster showed that the vast majority showed multiple highly conserved contacts (Campos et al., 2010). Each PulG protomer (P) within the fiber is involved in intricate contacts with protomers $\mathrm{P} \pm 1, \mathrm{P} \pm 3, \mathrm{P} \pm 4$ and possibly $\mathrm{P} \pm 7$ via different interfaces (Fig. 3). Biochemical analysis was 
used to test these contacts and validate the models, but also to probe the function of different interfaces. Site-directed mutagenesis and functional studies showed that the most extensive and important contacts involve the two closest protomers $(\mathrm{P}$ and $\mathrm{P} \pm 1)$. In the model of gonococcal T4a pilus, the key role in pilus assembly was attributed to electrostatic interactions between the absolutely conserved pilin residue $\mathrm{E} 5$ of protomer $\mathrm{P}$ and the $\mathrm{N}$-terminal amine group of the nearest protomer above $(\mathrm{P}+1)(\mathrm{Craig}$ et al., 2006). In the PulG pilus, in addition to this probable contact, which cannot be tested experimentally, two important salt bridge interactions have been identified involving negatively charged residues of the periplasmic $\alpha$-helical stem (E44 and D48) and positively charged residues (R87 and R88) in a loop region of the globular major pilin domain (Fig. 3). Highly conserved among the major pseudopilin family, these residues were essential for both T2SS functions: protein secretion and pseudopilus assembly (Campos et al., 2010). In contrast, mutations disrupting other interfaces differentially affect the two functions and appear to play different roles, currently under investigation (Nivaskumar, Campos et al, unpublished data).

The inter-protomer distances at the level of the hydrophobic pseudopilin segment have been tested by double cysteine cross-linking studies. As predicted by the pseudopilus structural models, the shortest distance in assembled pili is found between residues $10(\mathrm{P}+1)$ and $16(\mathrm{P})$. Placing Cys residues at these positions leads to covalent cross-linking of pili resulting in detergent-resistant PulG multimers. Interestingly, a lower degree of crosslinking is observed when cysteine residues are placed in neighboring positions, 9 and 16 on one hand, and 11 and 16 on the other. These mutually exclusive interactions suggest an iris-like movement of pilin monomers in assembled fibers (Campos et al., 2010). Similar structural heterogeneity is observed in archaella (Yu et al., 2012) and archeal pili (Wang et al., 2008) suggesting that other related fibers undergo similar conformational changes. External forces might promote these changes during assembly/disassembly and might propagate along the fibers, providing the 
173

174

175

176

177

178

179

180

181

182

183

184

185

186

187

188

189

190

191

192

193

194

195

structural basis for a potential signaling mechanism, one of the major functions observed in T4P.

\section{Minor pseudopilins}

Four low-abundance (minor) pseudopilins are essential for protein secretion under physiological expression conditions. In contrast, surface pili are produced, although less efficiently, in their absence when the components of the system are overproduced, leading to the idea that minor subunits provide determinants on the pseudopilus surface that are involved in secretion-specific interactions. In the simplest model, the secreted substrates interact directly with the minor pseudopilins. However, since complementation studies suggest that minor pseudopilins are not substrate specificity determinants \{Possot, $2000 \# 29$ \}, interactions with other T2SS factors and alternative functions such as the secretin channel gating, regulation of fiber length were proposed (Forest, 2008). Although extensive efforts were made to detect minor pseudopilins in surface fibers, there is still no biochemical or microscopy-based evidence for their co-assembly into pili in T2SS (Vignon et al., 2003). Nevertheless, they might be present in periplasmic pseudopili under physiological conditions, as suggested by studies of the Xanthomonas campestris T2SS (Kuo et al., 2005, Hu et al., 2002). In T4P, the equivalent minor pilins have been detected in pilus fractions and visualized by immuno-EM (Giltner et al., 2010, Winther-Larsen et al., 2005).

The most compelling argument for minor pseudopilin co-assembly into fibers is provided by the structural studies. The crystal structure of the complex formed by the periplasmic domains of EpsI and EpsJ from Vibrio cholerae indicated a 1-nm shift between the two proteins, identical to that in assembled pili (Yanez et al., 2008, Lam et al., 2009, Kohler et al., 2004). Furthermore, structural analysis of the GspJ-GspI-GspK trimer from enterotoxigenic 
E. coli showed that they formed a pilus-like complex (Korotkov and Hol, 2008) with a 1-nm axial rise and a rotational angle of $100^{\circ}$ between neighboring subunits. An alpha-helical domain of GspK inserted in the pilin $\beta$-sheet fold caps this complex, such that additional subunits cannot fit on top. Therefore, GspK is likely to be localized at the tip of a putative pseudopilus. Consistent with this model, the absolutely conserved residue E5 that is predicted to neutralize the N-terminal positive charge of the protomer above is lacking in GspK. The fourth minor pseudopilin $\mathrm{Xcp} \mathrm{U}^{\mathrm{GspH}}$ in $P$. aeruginosa interacts with the trimeric tip complex in vitro, suggesting that minor pseudopilins form a quaternary complex (Douzi et al., 2009). Based on extensive analysis of binding affinities between their periplasmic domains, Douzi et al proposed a pseduopilus assembly model in which $\mathrm{GspI}^{\mathrm{XcpV}}$ plays a central nucleating role and $\mathrm{GspH}^{\mathrm{XcpU}}$ links this complex to the major pseudopilin (\{Douzi, $\left.2009 \# 7\right\}$ In addition, structural modeling allowed fitting of EpsH subunit below the GspJ-GspI-GspK complex (Yanez et al., 2008), although direct binding of a GspG subunit also provided a satisfactory fit (Korotkov and Hol, 2008).

Early in vivo studies had suggested the role of GspI in assembly initiation (Sauvonnet et al., 2000b). Overproduction of PulK and its Pseudomonas homologue XcpX reduced pilus assembly, while deletion of $x c p X$ led to assembly of longer pili, leading to the idea that minor pseudopilins control the fiber length (Vignon et al., 2003, Durand et al., 2005). Recent systematic analysis of minor pseudopilin mutants by immunofluorescence microscopy revealed that not only pulK mutants, but also pulI and pulJ mutants make longer and fewer pili (Cisneros et al., 2011). Removing all minor pseudopilins led to complete loss of piliation, suggesting a role of the tip complex in pseudopilus assembly initiation. However, once initiated, pseudopilus assembly seems to continue unabated under plate culture conditions, as a function of the available pilin pool in the inner membrane. This observation further confirms the lack of active retraction and length control, consistent with the absence of retraction ATPase in the T2SS. 
223 The gspH mutants were identical to wild type cells with respect to pilus number, suggesting

224 that $\mathrm{GspH}$ does not play a role in initiation but rather at a later, elongation step, or in the 225 transition between initiation and elongation (Cisneros et al., 2012).

226 Mutants lacking all minor pseudopilins occasionally produced long pili, suggesting that

227 the assembly machinery spontaneously activates in rare cases and that minor pseudopilins play

228 a catalytic role. The minor pseudopilin pair GspJ-GspI is sufficient to restore a significant level

229 of piliation in these mutants, consistent with the central position of these proteins in the 230 quaternary pseudopilin complex (Cisneros et al., 2011, Douzi et al., 2009). In vivo they form a 231 staggered complex, as evidenced by close contacts between TM segment residues 10 (GspI) 232 and 16 (GspJ) shown by cysteine cross-linking (Cisneros et al., 2011). These contacts are 233 determined by a specific fit between the globular domains of these proteins, which form an 234 extensive interacting surface (Korotkov and Hol, 2008). Molecular dynamics simulations, 235 mutational and cross-linking studies provide evidence for self-assembly of tip pseudopilins: the staggered GspJ-GspI complex bends in the inner membrane to facilitate the binding of GspK alpha domain to its upper surface; this in turn facilitates the extraction of PulK, exerting a pulling force on the membrane. The strong correlation between GspJ-GspI-GspK binding and their ability to promote assembly initiation, suggests that these proteins transduce a signal to activate the assembly ATPase in the cytoplasm. How this is signal transduced and which protein-protein interactions participate in activation, are some of the major unresolved questions. Biochemical and structural studies from the Sandkvist and Hol labs in particular

243 provide some clues about the possible molecular mechanism of these events, discussed below.

\section{The pseudopilus assembly machinery}

247 The energy for the pseudopilus and T4P assembly is generated by conformational changes of 
the assembly ATPase of the GspE/PilB family. These hexameric ATPases form a ring-like structure with a central opening, leading initially to the idea that pilins are translocated to the periplasm via this channel (Camberg and Sandkvist, 2005). X-ray structures of EpsE from $V$. cholerae (Robien et al., 2003) and XpsE from X. campestris (Chen et al., 2005) show a bilobed monomer structure, with the ATP binding site at the hinge region between the N-terminal and C-terminal domains. In the recent EpsE hexamer model, the NTD and CTD of neighboring subunits share extensive contacts (Patrick et al., 2011). In this arrangement, which was tested by site-directed mutagenesis, several arginine residues around the active site belonging to both protomers seem to be required for ATP hydrolysis. Variants with alanine substitutions at these positions are specifically defective in ATP hydrolysis but not in ATP binding or GspE oligomerization. The strongest defect was associated with mutation R225A, in close proximity to the bound nucleotide both in $V$. cholerae EpsE and in $X$. campestris ATPase XspE, suggesting a direct role in ATP hydrolysis (Shiue et al., 2007).

In the hexamer, two neighboring subunits contribute to ATP binding and hydrolysis, resulting in three ATPase active sites (Patrick et al., 2011). Structural insight into their conformations comes from the studies of the closely related retraction ATPase PilT (Satyshur et al., 2007). Each PilT monomer pair shows a different active site conformation, 265 corresponding to the ATP-bound, hydrolysis and release state (Misic et al., 2010). Extensive rotation of the N-terminal domain around the active site hinge is involved in the transition

267 between the extreme states. Although these conformational states might appear stochastically, a 268 sequential model would be consistent with the biological data, suggesting that the addition of 269 one pseudopilin at a time at the level of the inner membrane drives both fiber polymerization and protein secretion (Campos et al., 2010). Interestingly, the subunit arrangement in the 271 initiation complex GspJ-GspI-GspK (Korotkov and Hol, 2008) mirrors the three-state ATPase model and might use the self-assembly energy or fit or induce the three-state ATPase motor 
273 (Cisneros et al., 2011), thus contradicting the three-start helix assembly model proposed for 274 T4P (Craig et al., 2006).

How are these conformational changes transduced to promote pseudopilus assembly?

One candidate for this role is GspF, the core fiber assembly component, whose interaction with

GspE has been demonstrated by two-hybrid and biochemical approaches (Py et al., 2001).

GspF has three TM segments and two large cytoplasmic domains structured as 6 -helix bundles (Abendroth et al., 2009). Little is known about the overall organization and oligomerization state of GspF. Its homologue in T4P, PilG, forms dimers and tetramers whose cryo-EM structure looks like a conical ring (Collins et al., 2007). Interactions of GspF of $P$. aeruginosa with GspL and GspE homologues are suggested by their mutual stabilization (Arts et al., 2007a) and by the yeast two-hybrid studies (Py et al., 2001). Details of these interactions and their functional significance are still a major black box.

The N-terminal domain of GspE forms a stable complex with the cytoplasmic domain of GspL, characterized by X-ray crystallography (Abendroth et al., 2005). GspL is the membrane anchor for the ATPase and favors its oligomerization. It also regulates the interactions with membrane acidic phospholipids, phosphatidyl glycerol and cardiolipin, which enhance GspE ATPase activity dramatically (Camberg et al., 2007).

Biochemical, two-hybrid and live imaging approaches provide ample evidence for interactions between GspL and GspM (Py et al., 2001, Possot et al., 2000, Buddelmeijer et al., 2006). In addition to its stabilizing effect on GspL, the C-terminal domain of GspM plays a specific role in secretion (Camberg et al., 2007). Recent studies on $V$. cholerae T2SS show that 294 EpsL and EpsG can be cross-linked in vivo (Gray et al., 2011), suggesting that EpsL transmits 295 the ATP-ase driven conformational changes to the major pseudopilin to promote fiber assembly. The GspL-GspG interacting surface requires further investigation, since epsG mutants defective in interaction with EpsL map at residues that form an intra-molecular 
hydrogen bond and affect the stability of EpsG monomer. Other T2SS components might interact with the major pseudopilin. In $N$. meningitidis, the major pilin subunit PilE interacts with PilG (a GspF homologue) and PilO, which is homologous to GspM (Georgiadou et al., 2012)

\section{Pseudopilus assembly and protein secretion}

T2SSs and T4P assembly systems are remarkably similar. Recent structural studies revealed that all T2SS components have their equivalents in T4aPS (Ayers et al., 2010). The minor pseudopilins, which are specifically required for protein secretion, have their counterparts in minor T4 pilins. Another "secretion specific" component GspM has a structural equivalent in the T4P PilO protein (Ayers et al., 2009).

Some components, like the prepilin peptidase, are functionally interchangeable between the T4P and T2SSs. The Pul T2SS can assemble the major T4 pilin PpdD encoded by the chromosome of E. coli and many other enterobacteria (Sauvonnet et al., 2000b, Kohler et al., 2004). Furthermore, the E. coli minor pilins can promote the initiation of PpdD assembly in a heterologous system, catalyzed by the T2SS assembly machinery (Cisneros et al., 2012), suggesting a role of minor T4 pilins in pilus assembly initiation. Minor T4 pilins are required for pilus assembly in the presence of retraction ATPase PilT. Mutants of pilT are piliated, due to the spontaneous assembly initiation events, the high concentration of pilins in the membrane and their extremely high stability. Indeed, unlike the T2SS pseudopili, which are unstable and short under physiological conditions, T4 pili are so stable that an energy-dependent process is required to undo the fibers and reset the system. While flexible modeling predicts a similar electrostatic and hydrophobic interaction network in pseudopili and T4P, the covalent disulfide 
322

323

bonds stabilizing the T4 pilin monomers might be responsible for this remarkable fiber stability (Campos et al., 2011, Li et al., 2012).

Interestingly, the minor T4 pilins of E. coli can promote the assembly of PulG, albeit less efficiently than the cognate minor pseudopilins (Cisneros et al., 2012). The absence of homology and therefore of structural complementarity between the globular domains of $\mathrm{T} 4$ pilins and the major T2SS pseudopilin (and vice versa) suggests that their role in assembly initiation does not rely on complex formation between these proteins. The only similarities between T4 pilins and T2SS pseudopilins are found in their hydrophobic segments, suggesting a role in crucial interactions with the assembly machinery. According to the activation model, the minor T4 pilin or minor pseudopilin complex formation acts upon the assembly machinery to start the first cycle of ATP binding and hydrolysis. However, pseudopilus assembly is more efficient in the presence of cognate minor pseudopilins, suggesting that a scaffolding mechanism (via direct binding of pseudopilin globular domains) contributes to assembly initiation (Cisneros et al., 2012, Cisneros et al., 2011, Burrows, 2012). In support of this model, full activation of pseudopilus assembly that leads to protein secretion is achieved in the presence of cognate pseudopilins in the T2SS.

\section{Models of protein secretion}

How is the energy of pseudopilus assembly transduced to promote protein secretion? Based on the similarities of T2SS and T4P, at least two models of protein secretion mechanism have been proposed (Nunn, 1999) known as "the piston" and the "Archimedes' screw" models. The idea of the piston was based on the dynamics of T4a pili, where cycles of pseudopilus extension and retraction push the substrate through the secretin channel. According to this model, pseudopilus assembly provides the driving force for secretion, acting as a piston, while 
minor pseudopilins determine the binding specificity (Shevchik et al., 1997, Nunn, 1999, Vignon et al., 2003). Major support for the piston model comes from recent findings that the minor pseudopilin complex $\mathrm{XcpU}-\mathrm{V}-\mathrm{W}-\mathrm{X}$ of $P$. aeruginosa binds in vitro to the specific substrate of the Xcp system, LasB, but not to the substrate of the second Hxc system present in the same species (Douzi et al., 2011).

The major problem with this model is that in T2SS there is no evidence of fiber retraction. Certain T4P assembly systems also secrete periplasmic proteins. One example is the toxin co-regulated pilus (TCP) of $V$. cholerae, which secretes a soluble colonization factor TcpF (Kirn et al., 2003). Interestingly, the TCP system lacks the retraction ATPase, again suggesting that pilus retraction is not required for protein secretion. A unidirectional assembly might be required to ensure the vectorial transfer of proteins from the inner membrane towards the exterior. The lack of retraction ATPase in T2SS led to the proposal that pseudopilus "collapses" or is degraded, and that GspK might catalyze the degradation of major pseudopilin (Durand et al., 2005). Indeed, pseudopilins might be more prone to degradation than T4 pilins. Their $\mathrm{Ca}^{2+}$-coordinating C-terminal loop, structurally equivalent to the disulfide loop in $\mathrm{T} 4$ pilins, might provide a basis for the control of subunit structural integrity (Korotkov et al., 2009).

In T4P and all helical fibers of this superfamily, subunits are shifted by a 1-nm translation and variable rotation angles, with a symmetry that does not match that of the GspE ATPase. This implies that, during assembly, the fibers rotate relative to the assembly ATPase during addition of each new subunit (Mattick, 2002). The T2SS might be regarded as a modified reverse ATP synthase that uses the energy release upon ATP hydrolysis for rotation instead of generating ATP using the proton motive force (Streif et al., 2008). The Archimedes' screw model postulates exoprotein binding to the pseudopilus at the base, suggesting that major pseudopilins provide the binding surface (Fig. 4). Addition of pseudopilin subunits would be 
coupled to the rotary motion of the fiber and to a net upward transfer of subunits and bound exoproteins from the pseudopilus base into the secretin channel. So far, there is less biochemical support for this model that for the piston model. However, the exoprotein PulA of the K. oxytoca T2SS interacts with the PulG pili (Kohler et al., 2004) (Francetic et al, submitted). In addition, major pseudopilin mutations lead to a change in substrate specificity in Xcp and Hxc, the two T2SS of $P$. aeruginosa (Durand et al., 2011).

Evidence for rotation during or upon assembly of helical fibers comes from archaella, which rotate to promote swimming (Ghosh and Albers, 2011, Herzog and Wirth, 2012); S.V. Albers, personal communication). While bacterial flagella require proton motive force for rotation, archaella use the energy of ATP hydrolysis and do not seem to require motor proteins other than the assembly machinery itself (Streif et al., 2008). Given their conservation, it is plausible that all helical fiber assembly nanomachines of this superfamily display the same dynamics. While rotation of rigid archaella allows them to "carry the weight" of the archaeal cell and support swimming, rotation of flexible pseudopili or T4P might not be easily observed. The ATPase GspE in T2SS is anchored to the membrane via GspL, but GspF might be free to rotate in the membrane. Components of the fiber assembly platform anchored in the cell wall might play a role of the stator, and the link with GspC and GspD might be important in this regard (Korotkov et al., 2011).

Clearly, the interactions between the membrane components of fiber assembly nanomachines and fiber dynamics are a vast unexplored area that awaits further research. Comparative analysis of T2SSs, T4P, archaeal pili and archaella should provide important clues on the common mechanisms and significant differences that underlie the specialized functions of these remarkable systems.

\section{Acknowledgments}


The study of protein secretion in our team has been funded by the Institut Pasteur grant PTR339. MC was a scholar of the French Ministry of Science and Education, MN is the recipient of Pasteur-Paris University fellowship and DC was funded by EMBO long term and Roux fellowships. We are grateful to our collaborators Michaël Nilges, Guillaume Bouvier,

Peter J. Bond, Gérard Péhau-Arnaudet, Nadia Izadi-Pruneyre, Daniel Ladant and Gouzel

Karimova for their contributions to the pseudopilus project. We are grateful to all members of the Molecular Genetics Unit for many stimulating discussions. We thank Tony Pugsley for generous support and for the critical reading of the manuscript.

\section{References}

Abendroth, J., D. Mitchell, K. Korotkov, T. Johnson, A. Kreger, M. Sankvist and W. Hol, 2009. The three-dimensional structure of the cytoplasmic domains of EpsF from the type 2 secretion system of Vibrio cholerae. J Struct Biol 166, 303-315.

Abendroth, J., P. Murphy, M. Sandkvist, M. Bagdasarian and W. G. Hol, 2005. The X-ray structure of the type II secretion system complex formed by the N-terminal domain of EpsE and the cytoplasmic domain of EpsL of Vibrio cholerae. J Mol Biol 348, 845-855.

Albers, S. V. and M. Pohlschroder, 2009. Diversity of archaeal type IV pilin-like structures. Extremophiles 13, 403-410.

Alphonse, S., E. Durand, B. Douzi, B. Waegele, H. Darbon, A. Filluox, R. Voulhoux and C. Bernard, 2010. Structure of the Pseudomonas aeruginosa XcpT pseudopilin, a major component of the type II secretion system. J. Struct. Biol. 169, 75-80.

Arts, J., A. de Groot, G. Ball, E. Durand, M. El Khattabi, A. Filloux, J. Tommassen and M. Koster, 2007a. Interaction domains in the Pseudomonas aeruginosa type II secretory apparatus component XcpS (GspF). Microbiol 153, 1582-1592.

Arts, J., R. van Boxtel, A. Filloux, J. Tommassen and M. Koster, 2007b. Export of the pseudopilin XcpT of the Pseudomonas aeruginosa type II secretion system via the signal recognition particle-Sec pathway. J Bacteriol. 189, 2069-2076.

Ayers, M., P. L. Howell and L. L. Burrows, 2010. Architecture of the type II secretion and type IV pilus machineries. Future Microbiol 5, 1203-1218.

Ayers, M., L. Sampaleanu, S. Tammam, J. Koo, H. Harvey, P. Howell and L. Burrows, 2009. $\mathrm{PilM} / \mathrm{N} / \mathrm{O} / \mathrm{P}$ proteins form an inner membrane compex that affects the stability of the Pseudomonas aeruginosa type IV pilus secretin. J Mol. Biol. 394, 128-142.

Buddelmeijer, N., O. Francetic and A. P. Pugsley, 2006. Green fluorescent chimeras indicate nonpolar localization of pullulanase secreton components PulL and PulM. J. Bacteriol.188, 2928-2935.

Burrows, L. L., 2012. Prime time for minor subunits in type II secretion and type IV pilus systems. Mol Microbiol 86.

Camberg, J. L., T. L. Johnson, M. Patrick, J. Abendroth, W. G. Hol and M. Sandkvist, 2007. Synergistic stimulation of EpsE ATP hydrolysis by EpsL and acidic phospholipids. EMBO J 26, 19-27. 
Camberg, J. L. and M. Sandkvist, 2005. Molecular analysis of the Vibrio cholerae type II secretion ATPase EpsE. J Bacteriol 187, 249-256.

Campos, M., O. Francetic and M. Nilges, 2011. Modeling pilus strcutures from sparse data. J J Struct Biol. 173, 436-444.

Campos, M., M. Nilges, D. A. Cisneros and O. Francetic, 2010. Detailed structural and assembly model of the type II secretion pilus from sparse data. Proc Natl Acad Sci USA 107, 13081-13086.

Carbonnelle, E., S. Helaine, X. Nassif and V. Pelicic, 2006. A systematic genetic analysis in Neisseria meningitidis defines the Pil proteins required for assembly, functionality, stabilization and export of type IV pili. Mol Microbiol 61, 1510-1522.

Chen, Y., S. J. Shiue, C. W. Huang, J. L. Chang, Y. L. Chien, N. T. Hu and N. L. Chan, 2005. Structure and function of the XpsE N-terminal domain, an essential component of the Xanthomonas campestris type II secretion system. J Biol Chem 280, 42356-42363.

Cisneros, D., G. Péhau-Arnaudet and O. Francetic, 2012. Heterologous assembly of type IV pili by a type II secretion system reveals the role of minor pilins in assembly initiation. Mol Microbiol 86.

Cisneros, D. A., P. J. Bond, A. P. Pugsley, M. Campos and O. Francetic, 2011. Minor pseudopilin self-assembly primes type II secretion pseudopilus elongation. EMBO J 31, 1041-1053.

Collins, R. F., M. Saleem and J. P. Derrick, 2007. Purification and three-dimensional electron microscopy structure of the Neisseria meningitidis Type IV pilus biogenesis protein PilG. J Bacteriol 189, 6389-6396.

Craig, L., R. K. Taylor, M. E. Pique, B. D. Adair, A. S. Arvai, M. Singh, S. J. Lloyd, D. S. Shin, E. D. Getzoff, M. Yeager, F. K.T. and J. A. Tainer, 2003. Type IV pilin structure and assembly: X-ray and EM analyses of Vibrio cholerae toxin-coregulated pilus and Pseudomonas aeruginosa PAK pilin. Molec Cell 11, 1139-1150.

Craig, L., N. Volkmann, A. S. Arvai, M. E. Pique, M. Yeager, E. H. Egelman and J. A. Tainer, 2006. Type IV pilus structure by cryo-electron microscopy and crystallography: implications for pilus assembly and functions. Mol Cell 23, 651-662.

Douzi, B., G. Ball, C. Cambillau, M. Tegoni and R. Voulhoux, 2011. Deciphering the Xcp Pseudomonas aeruginosa type II secretion machinery through multiple interactions with substrates. J Bio Chem 286, 40792-40801.

Douzi, B., E. Durand, C. Bernard, S. Alphonse, C. Cambillau, A. Filloux, M. Tegoni and R. Voulhoux, 2009. The XcpV/GspI pseudopilin has a central role in the assembly of a quaternary complex within the T2SS pseudopilus. J Biol Chem 284, 34580-34589.

Douzi, B., A. Filloux and R. Voulhoux, 2012. On the path to uncover the bacterial type II secretion system. Phil Trans R Soc B 367, 1059-1072.

Durand, E., S. Alphonse, C. Brochier-Armanet, G. Ball, B. Douzi, A. Filloux, C. Bernard and R. Voulhoux, 2011. The assembly mode of the pseudopilus. A hallmark to distinguish a novel secretion system subtype. J. Biol. Chem. 286, 24407-24416.

Durand, E., A. Bernadac, G. Ball, A. Lazdunski, J. N. Sturgis and A. Filloux, 2003. Type II protein secretion in Pseudomonas aeruginosa: the pseudopilus is a multifibrillar and adhesive structure. J Bacteriol 185, 2749-2758.

Durand, E., G. Michel, R. Voulhoux, J. Kurner, A. Bernadac and A. Filloux, 2005. XcpX controls biogenesis of the Pseudomonas aeruginosa XcpT-containing pseudopilus. The J Biol Chem 280, 31378-31389.

Forest, K. T., 2008. The type II secretion arrowhead: the structure of GspI-GspJ-GspK. Nat Struct Mol Biol 15, 428-430. 
Francetic, O., N. Buddelmeijer, S. Lewenza, C. A. Kumamoto and A. P. Pugsley, 2007. Signal recognition particle-dependent inner membrane targeting of the PulG pseudopilin component of a type II secretion system. J Bacteriol 189, 1783-1793.

Georgiadou, M., M. Castagnini, G. Karimova, D. Ladant and V. Pelicic, 2012. Large-scale study of the interactions between proteins involved in type IV pilus biology in Neisseria meningitidis: characterization of a sub-complex involved in pilus assembly. Mol Microbiol 84, 857-873.

Ghosh, A. and S. V. Albers, 2011. Assembly and function of the archaeal flagellum. Biiochem Soc Trans 39, 64-69.

Giltner, C. L., M. Habash and L. L. Burrows, 2010. Pseudomonas aeruginosa minor pilins are incorporated into type IV pili. J Mol Biol 398, 444-461.

Gray, M. D., M. Bagdasarian, W. G. Hol and M. Sandkvist, 2011. In vivo cross-linking of EpsG to EpsL suggests a role for EpsL as an ATPase-pseudopilin coupling protein in the Type II secretion system of Vibrio cholerae. Mol Microbiol 79, 786-798.

Herzog, B. and R. Wirth, 2012. Swimming behaviour of selected species of archea. Appl Environ Micribiol. 78, 1670-1674.

Hobbs, M. and J. S. Mattick, 1993. Common components in the assembly of type 4 fimbriae, DNA transfer systems, filamentous phage and protein-secretion apparatus: a general system for the formation of surface-associated protein complexes. Mol. Microbiol. 10, 233-243.

Hu, N. T., W. M. Leu, M. S. Lee, A. Chen, S. C. Chen, Y. L. Song and L. Y. Chen, 2002. $\mathrm{XpsG}$, the major pseudopilin in Xanthomonas campestris pv. campestris, forms a piluslike structure between cytoplasmic and outer membranes. Biochem. J 365, 205-211.

Jarrel, K. F. and S. V. Albers, 2012. The archeallum: an old motility structure with a new name. Trends Microbiol 20, 307-312.

Kirn, T. J., N. Bose and R. K. Taylor, 2003. Secretion of a soluble colonization factor by the TCP type 4 pilus biogenesis pathway in Vibrio cholerae. Mol. Microbiol. 49, 81-92.

Kohler, R., K. Schafer, S. Muller, G. Vignon, K. Diederichs, A. Philippsen, P. Ringler, A. P. Pugsley, A. Engel and W. Welte, 2004. Structure and assembly of the pseudopilin PulG. Mol Microbiol 54, 647-664.

Korotkov, K., T. L. Johnson, M. Jobling, J. Pruneda, E. Pardon, A. Héroux, S. Turley, J. Steayert, R. Holmes, S. M and H. WG, 2011. Structural and functional studies on the inetractions of GspC and GspD in type II secretion system. PLOS Pathog.

Korotkov, K., M. Sandkvist and W. Hol, 2012. The type II secretion system: biogenesis, molecular archtecture and mechanism. Nat Rev Microbiol 10, 336-351.

Korotkov, K. V., M. Gray, A. Kreger, S. Turley, M. Sandkvist and W. Hol, 2009. Calcium is essential for the major pseudopilin in the type 2 secretion system. J Biol Chem 284, 25466-25470.

Korotkov, K. V. and W. G. Hol, 2008. Structure of the GspK-GspI-GspJ complex from the enterotoxigenic Escherichia coli type 2 secretion system. Nat Struct Mol Biol 15, 462468.

Kuo, W. W., H. W. Kuo, C. C. Cheng, H. L. Lai and L. Y. Chen, 2005. Roles of the minor pseudopilins, XpsH, XpsI and XpsJ, in the formation of XpsG-containing pseudopilus in Xanthomonas campestris pv. campestris. J Biomed Sci 12, 587-599.

Lam, A. Y., E. Pardon, K. V. Korotkov, W. G. Hol and J. Steyaert, 2009. Nanobody-aided structure determination of the EpsI:EpsJ pseudopilin heterodimer from Vibrio vulnificus. J Struct Biol 166, 8-15.

Li, J., E. Egelman and L. Craig, 2012. Structure of the VIbrio choleare type IV b pilus and stability comparison with Neisseria gonorrhoeae type IV a pilus. J Mol Biol 418, 47-64.

Mattick, J. S., 2002. Type IV pili and twitching motility. Annu Rev Microbiol 56, 289-314. 
McLaughlin, L., R. Haft and F. KT, 2012. Structural insights into the Type II secretion nanomachine. $\mathrm{Cu}$ Op Struct Biol 22, 208-216.

Misic, A. M., K. A. Satyshur and K. T. Forest, 2010. P. aeruginosa PilT structure with and without nucleotide reveal a dynamic type IV pilus retraction motor. J Mol Biol 400, 1011-1021.

Nunn, D., 1999. Bacterial type II protein export and pilus biogenesis:more than just homologies. Trends Cell Biol 9, 402-408.

Patrick, M., K. V. Korotkov, W. G. Hol and M. Sandkvist, 2011. Oligomerization of EpsE coordinates residues from multiple subunits. J BIol. Chem 286, 10378-10386.

Pelicic, V., 2008. Type IV pili: e pluribus unum? Mol Microbiol 68, 827-837. Possot, O. M., G. Vignon, N. Bomchil, F. Ebel and A. P. Pugsley, 2000. Multiple interactions between pullulanase secreton components involved in stabilization and cytoplasmic membrane association of PulE. J Bacteriol 182, 2142-2152.

Pugsley, A. P., 1993. Processing and methylation of PulG, a pilin-like component of the general secretory pathway of Klebsiella oxytoca. Mol Microbiol 9, 295-308.

Py, B., L. Loiseau and F. Barras, 2001. An inner membrane platform in the type II secretion machinery of Gram-negative bacteria. EMBO Rep 2, 244-248.

Robien, M. A., B. E. Krumm, M. Sandkvist and W. G. Hol, 2003. Crystal structure of the extracellular protein secretion NTPase EpsE of Vibrio cholerae. J Mol. Biol 333, $657-$ 674.

Satyshur, K. A., G. A. Worzalla, L. S. Meyer, E. K. Heiniger, K. G. Aukema, A. M. Misic and K. T. Forest, 2007. Crystal structures of the pilus retraction motor PilT suggest large domain movements and subunit cooperation drive motility. Structure 15, 363-376

Sauvonnet, N., P. Gounon and A. P. Pugsley, 2000a. PpdD type IV pilin of Escherichia coli K12 can be assembled into pili in Pseudomonas aeruginosa. J Bacetriol. 182, 848-854.

Sauvonnet, N., G. Vignon, A. P. Pugsley and P. Gounon, 2000b. Pilus formation and protein secretion by the same machinery in Escherichia coli. EMBO J 19, 2221- 2228.

Shevchik, V., J. Robert-Baudouy and G. Condemine, 1997. Specific interaction between OutD, an Erwinia chrysanthemi outer membrane protein of the general secretory pathway, and secreted proteins. EMBO J 16, 3007-3016.

Shiue, S. J., I.-L. Chien, N. L. Chan, W. M. Leu and N. T. Hu, 2007. Mutation of a key residue in the type II secretion system ATPse uncouples ATP hydrolysis from protein translocation. Mol Microbiol 65, 401-412.

Streif, S., W. F. Staudinger, W. Marwan and D. Oesterhelt, 2008. Flagella rotation in the archaeon Halobacterium salinarum depends on ATP. J Mol Biol 384, 1-8.

Strom, M. S. and S. Lory, 1991. Amino acid substitutions in pilin of Pseudomonas aeruginosa. Effect on leader peptide cleavage, amino-terminal methylation, and pilus assembly. J. Biol. Chem. 266, 1656-1664.

Vignon, G., R. Kohler, E. Larquet, S. Giroux, M. C. Prevost, P. Roux and A. P. Pugsley, 2003. Type IV-like pili formed by the type II secreton: specificity, composition, bundling, polar localization, and surface presentation of peptides. J Bacteriol 185, 3416-3428.

Wang, Y. A., X. Yu, S. Y. M. Ng, K. F. Jarrell and E. H. Egelman, 2008. The structure of an archaeal pilus. J Mol Biol 381, 456-466.

Winther-Larsen, H. C., M. Wolfgang, S. Dunham, J. P. van Putten, D. Dorward, C. Lovold, F. E. Aas and M. Koomey, 2005. A conserved set of pilin-like molecules controls type IV pilus dynamics and organelle-associated functions in Neisseria gonorrhoeae. Mol Microbiol 56, 903-917.

Wolfgang, M., J. P. M. van Putten, S. F. Hayes, D. Dorward and M. Koomey, 2000. Components and dynamics of fiber formation define a ubiquitous biogenesis pathway for bacterial pili. EMBO. J. 19, 6408-6418. 
Yanez, M., K. Korotkov, J. Abendroth and W. G. J. Hol, 2008. The Crystal Structure of a B inary Complex of two Pseudopilins: EpsI and EpsJ from the Type 2 Secretion System of Vibrio vulnificus. J. Mol. Biol. 375, 471-486.

Yanez, M. E., K. V. Korotkov, J. Abendroth and W. G. Hol, 2008. Structure of the minor pseudopilin EpsH from the Type 2 secretion system of Vibrio cholerae. J. Mol Biol 377, 91-103.

Yu, X., C. Goforth, C. Meyer, R. Reinhard, R. Wirth, G. F. Shchröder and E. H. Egelman, 2012. Filaments from Ignicoccis hospitalis show diversity of packing in proteins containing N-terminal type IV pilin helices. J Mol Biol 422, 264-281. 
602

603

604

Figures

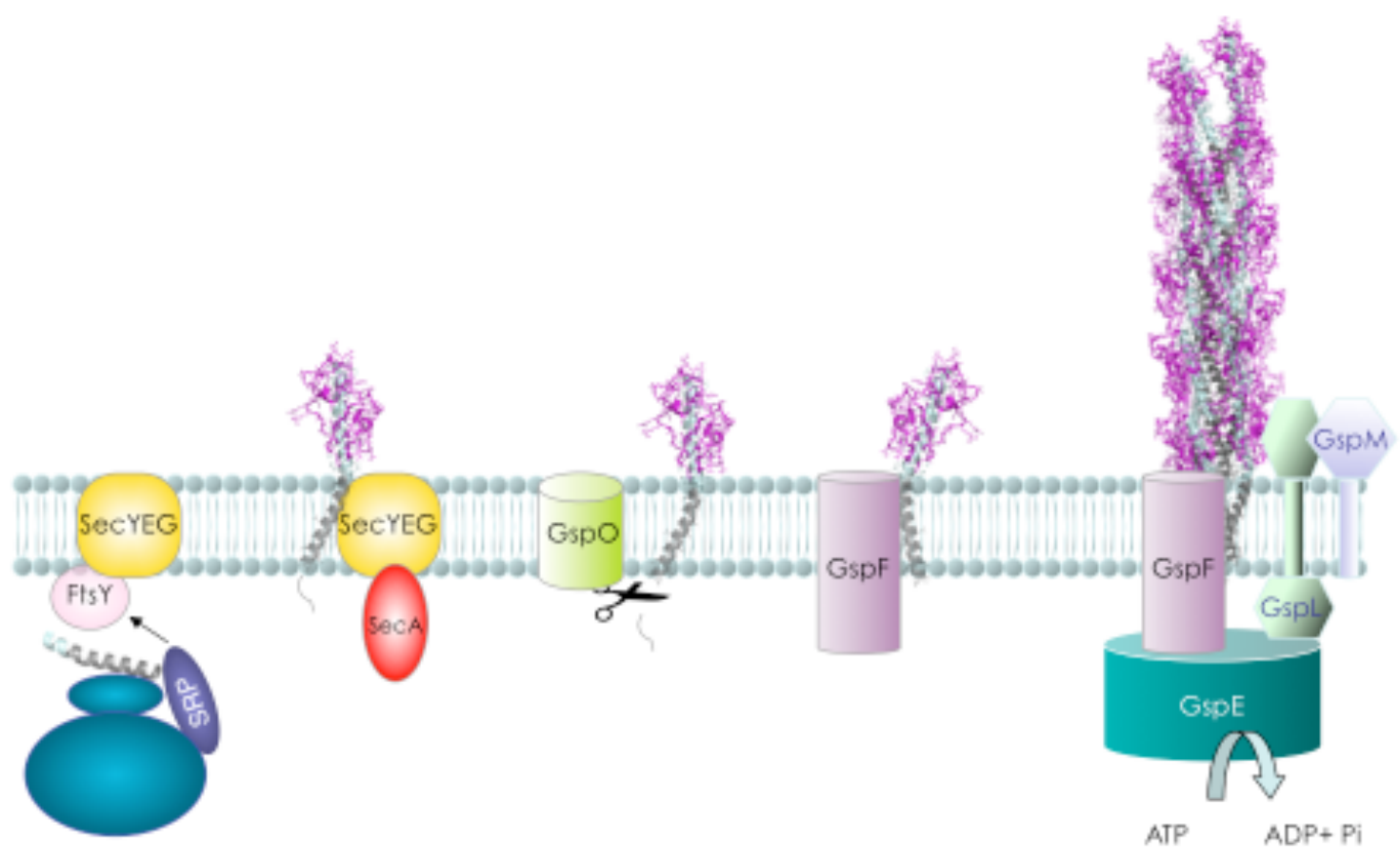

$\begin{array}{lllll}\text { A } & \text { B } & \text { C } & \text { D } & \text { E }\end{array}$

605

606

607

608

609

610

611

612

613

614

615

616

617

618
Fig. 1. Pseudopilus biogenesis steps. The transmembrane segment of pseudopilins (in grey) interacts with cellular and type II secretion system partners. The hydrophobicity of the segment is crucial for the targeting via the SRP complex (A) to the Sec translocase (B) for correct membrane insertion. Upon insertion, the prepilin peptidase cleaves the positively charged prepeptide after the conserved Gly residue (C); the cleaved peptide interacts with at least one component of the assembly platform (D) to finally become buried in the core of the assembled fiber, while the globular hydrophilic domains are exposed on the pseudopilus surface (E). 

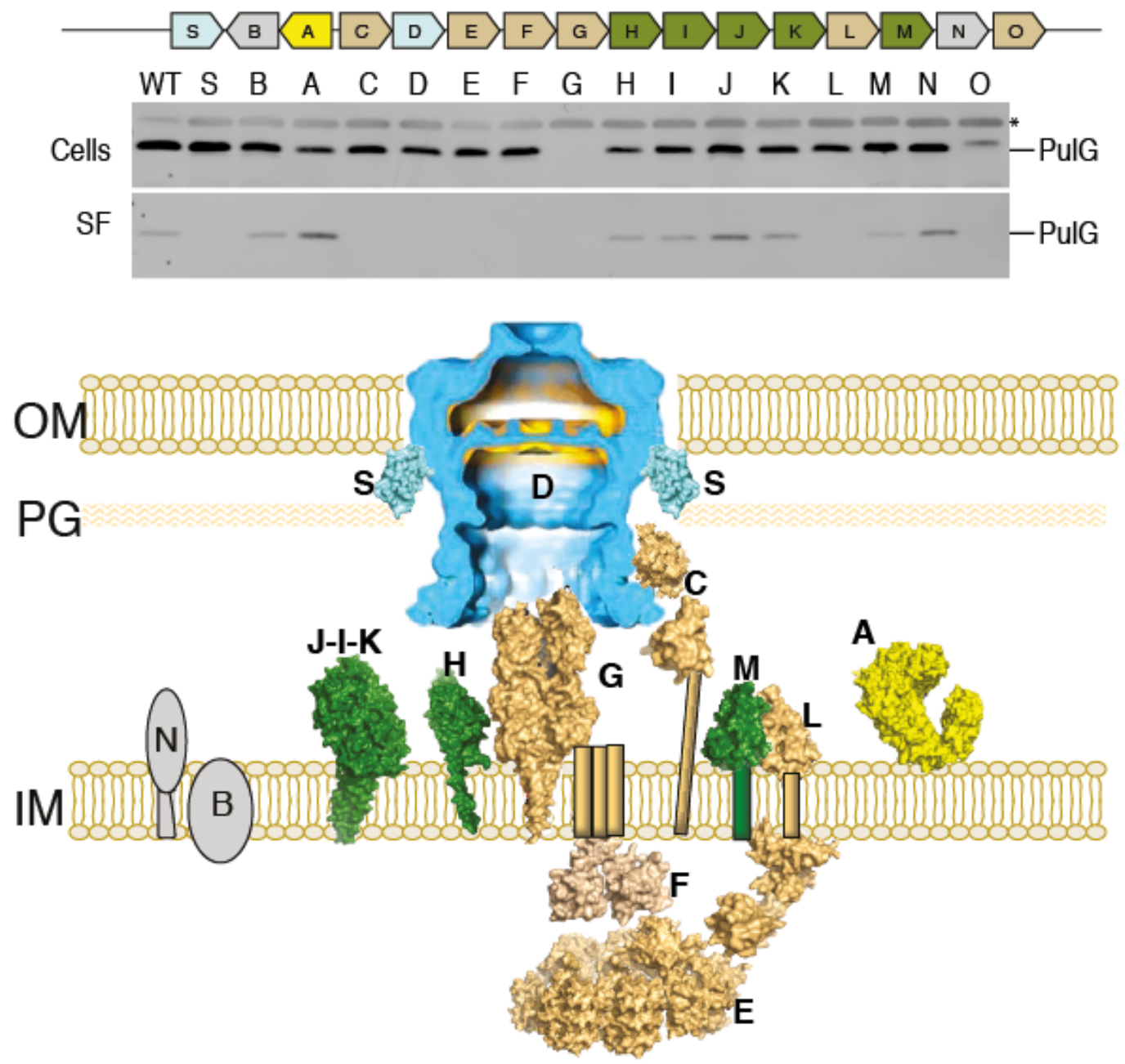

620 Fig. 2. Involvement of T2SS components in pilus formation. (A) Top: Organization of the Pul T2SS-encoding genes on the chromosome of Klebsiella oxytoca. Divergent operons pulAB and pulCDEFGHIJKLMNO, and a monocystronic pulS gene encode the T2SS. The substrate gene $\mathrm{A}$ is shown in yellow. Genes dispensable for the substrate secretion are shown in grey, genes essential for surface exposure of the pseudopilus and the substrate are in blue, genes essential for pseudopilus assembly and protein secretion are in pale orange, and genes essential for protein secretion only are in green. Below: PulG pilus assembly in mutants lacking individual T2SS components. Bacteria of strain MDS42 (Posfai et al., 2006) transformed with different derivatives of plasmid pCHAP231 carrying the complete set of pul genes or single nonpolar mutations as indicated (Possot et al., 2000) were grown for two days on rich solid media. Bacterial and sheared pili fractions were analyzed by immunodetection using anti-PulG antibodies, as described (Cisneros et al., 2011). The equivalent of $0.05 \mathrm{OD}_{600 \mathrm{~nm}}$ of each fraction was loaded. The asterisk indicates a cross-reacting band. (B) The schematic representation of the Pul T2SS components in the bacterial envelope with the same color- code as above. OM, outer membrane; PG, peptidoglycan; IM, inner membrane. Images of T2SS components were prepared with PyMol (http://www.pymol.org). The model of GspD is adapted from (Reichow et al., 2010). 
641 642 643 644 645 646 647 648 649 650 651 652
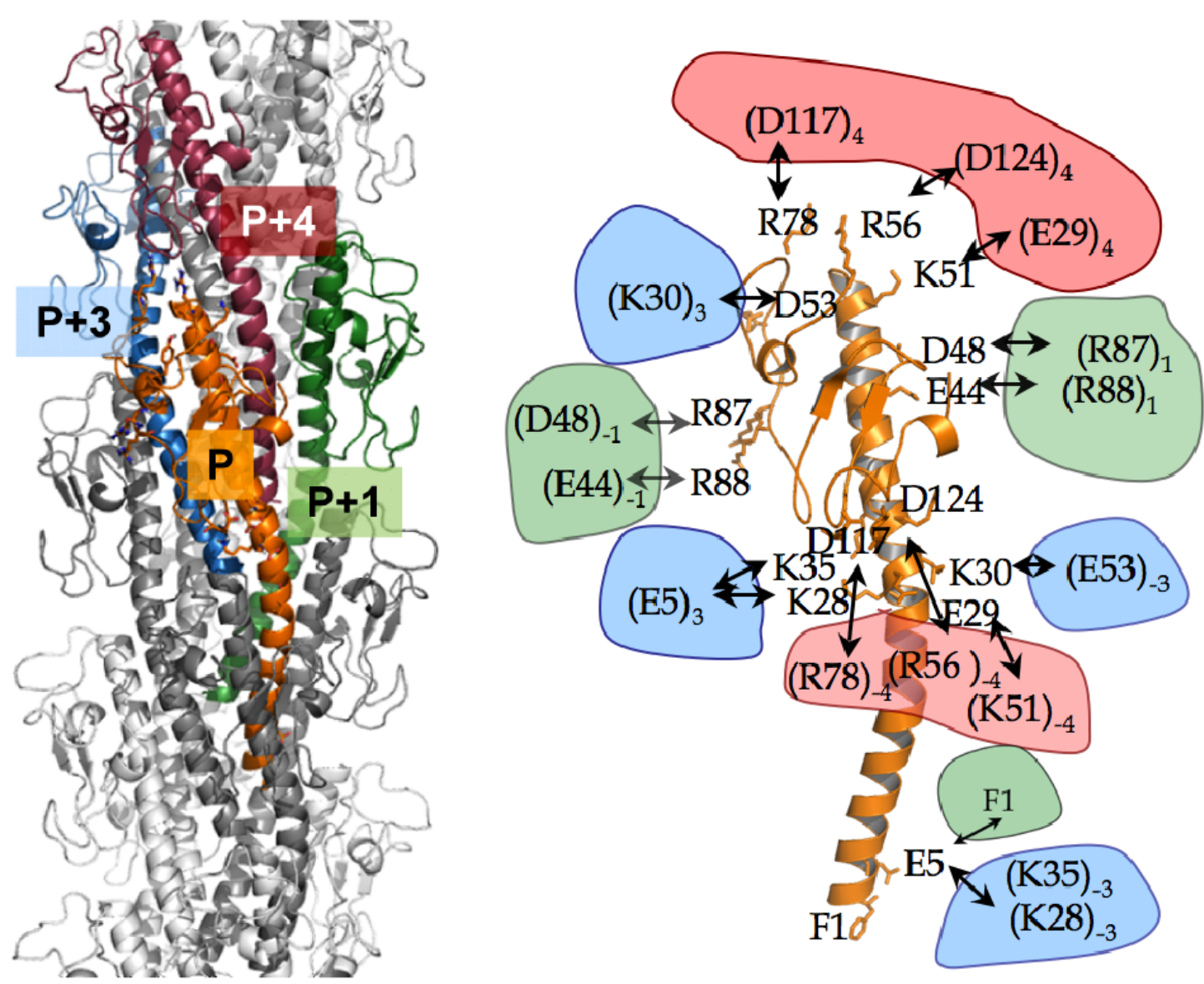

Fig. 3. The structure of the PulG pseudopilus (left) and PulG residues involved in interprotomer interactions (right). The central protomer $\mathrm{P}$ (orange) interacts with protomer $\mathrm{P}+1$ (green), $\mathrm{P}+3$ (blue) and $\mathrm{P}+4$ (red). Arrows indicate predicted interactions between residues of the central protomer $\mathrm{P}$ and its partners at each interface (indicated by the residue position, the color code and the protomer number in index). Adapted from (Campos et al., 2010). 
A
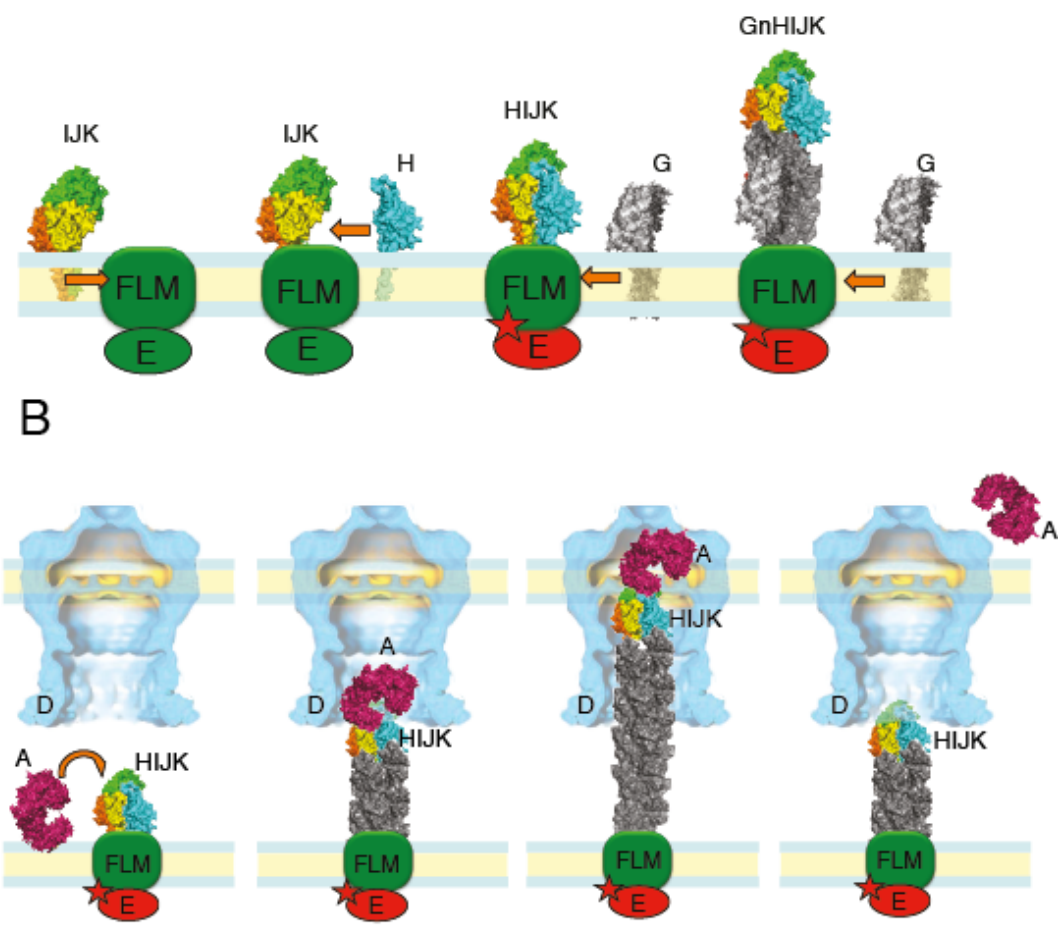

C

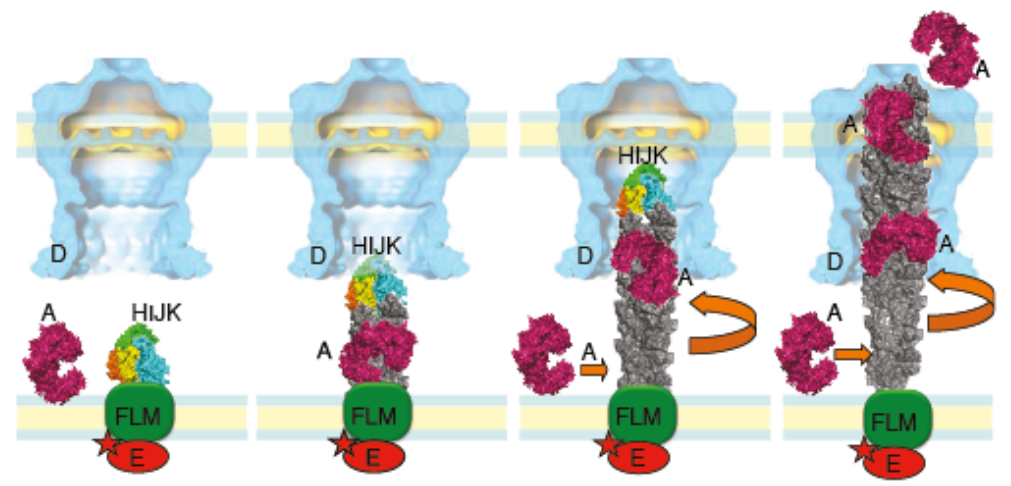

654 Fig. 4. Models of pseudopilus assembly and function in protein secretion. (A) The minor pseudopilins (I-J-K) self-assemble in the inner membrane (IM) and bind to the fourth minor pseudopilin $\mathrm{GspH}(\mathrm{H})$. This tetrameric complex transfers the activation signal to the assembly ATPase via an unknown component of the assembly platform, possibly GspL (LMF). The activation of the ATPase (E) leads to the elongation of the pseudopilin by successive addition of major pseudopilin subunits $(\mathrm{G})$, coupled to the fiber rotation relative to the assembly ATPase. (B) According to the piston model, the substrate (A in pink) binds to the minor pseudopilin complex on the tip (HIJK). Pseudopilus elongation adding GspG subunits (in grey) pushes the substrate through the secretin channel. The system is reset by an unknown mechanism to allow binding of the next substrate molecule. (C)In the Archimedes' screw model, minor pseudopilin complex initiates pseudopilus assembly and the substrate binds to the major pseudopilins at the fiber base in the IM. Pseudopilus growth from the base, coupled with rotation drives the substrate into the secretin channel (D) vestibule. Pseudopilus assembly force pushes the secretin gate open and the substrate is expelled across the outer membrane (OM) channel. A single pseudopilus initiation event leads to secretion of multiple substrate molecules via continuous assembly of the major pseudopilins. 